\title{
THE POINCARÉ ALGEBRA IN THE CONTEXT OF AGEING SYSTEMS: LIE STRUCTURE, REPRESENTATIONS, APPELL SYSTEMS AND COHERENT STATES
}

\author{
MALTE HENKEL \\ Groupe de Physique Statistique, \\ Institut Jean Lamour (CNRS UMR 7198), \\ Université de Lorraine Nancy, B.P. 70239, \\ F - 54506 Vandouvre-lès-Nancy Cedex, France \\ malte.henkel@ijl.nancy-universite.fr \\ RENÉ SCHOTT \\ Institut Élie Cartan (IECN - CNRS UMR 7502), \\ LORIA (CNRS UMR 7503), Université de Lorraine Nancy, \\ B.P. 70239, F-54506 Vandœuvre-lès-Nancy Cedex, France \\ rene.schott@loria.fr \\ STOIMEN STOIMENOV \\ Institute of Nuclear Research and Nuclear Energy, \\ Bulgarian Academy of Sciences, \\ $B G-1784$ Sofia, Bulgaria \\ spetrov@inrne.bas.bg \\ JÉRÉMIE UNTERBERGER \\ Institut Élie Cartan (IECN - CNRS UMR 7502), \\ Université de Lorraine Nancy, B.P. 70239, \\ F - 54506 Vandouvre-lès-Nancy Cedex, France \\ jeremie.unterberger@inria.fr \\ Received 16 July 2012 \\ Revised 22 October 2012 \\ Accepted 22 October 2012 \\ Published 31 December 2012
}

\begin{abstract}
By introducing an unconventional realization of the Poincaré algebra $\mathfrak{a l t}_{1}$ of special relativity as conformal transformations, we show how it may occur as a dynamical symmetry algebra for ageing systems in non-equilibrium statistical physics and give some applications, such as the computation of two-time correlators. We also discuss infinitedimensional extensions of $\mathfrak{a l t}_{1}$ in this setting. Finally, we construct canonical Appell systems, coherent states and Leibniz function for $\mathfrak{a l t}_{1}$ as a tool for bosonic quantization.
\end{abstract}

Keywords: Poincaré algebra; conformal Galilean algebra; central extension; nonequilibrium dynamical scaling; Appell systems.

AMS Subject Classification: 22E46, 53C35, 57S20 


\section{Introduction}

Ageing phenomena occur widely in physics: glasses, granular systems or phaseordering kinetics are just a few examples, see e.g. [5, 9, 23, 24] for reviews. Ageing phenomena may typically arise in the presence of two competing, globally equivalent, steady-states: except for very particular initial preparations, the physical system does not relax to any of these; rather there appear ordered domains which grow in size with time and which are separated by fluctuating boundaries. ${ }^{\text {a }}$ A convenient way to describe this sort of system is through a Langevin equation, which might schematically be written as

$$
2 \mathcal{M} \partial_{t} \phi=-\frac{\delta \mathcal{F}}{\delta \phi}+\eta
$$

where $\phi=\phi(t, \mathbf{r})$ stands for the physical order-parameter (here assumed to be nonconserved), $\mathcal{F}$ is the Ginzburg-Landau functional, the "mass" $\mathcal{M}$ plays the role of a kinetic coefficient and $\eta$ describes a Gaussian, delta-correlated noise. While it is well-accepted [5] that systems of this kind should display some sort of dynamical scaling when brought into the situation sketched above, the question has been raised whether their non-equilibrium dynamics might possess more symmetries than merely scale-invariance [19]. At first sight, the noisy terms in the Langevin equation (1.1) might appear to exclude any nontrivial answer. However, a more refined answer is possible. One may consider (1.1) as the classical equation of motion of an associated field-theory, whose action reads

$$
S[\phi, \widetilde{\phi}]=S_{0}[\phi, \widetilde{\phi}]+S_{b}[\widetilde{\phi}]
$$

where $\widetilde{\phi}$ is the response-field associated to the order-parameter field $\phi$. Here, the "noise" as described by the random force $\eta$ only enters into the second term $S_{b}[\widetilde{\phi}]$. In many cases, the so-called "noise-less" part $S_{0}$ takes a free-field form

$$
S_{0}[\phi, \widetilde{\phi}]=\int \mathrm{d} t \mathrm{~d} \mathbf{r} \widetilde{\phi}\left(2 \mathcal{M} \partial_{t}-\Delta\right) \phi
$$

which has the important property of being Galilei-invariant. If that is the case, the Bargman superselection rules coming from the Galilei-invariance of $S_{0}$ allow one to show that all $n$-point correlation and response functions of the theory can be expressed in terms of certain $(n+2)$-point correlation function of an effective deterministic theory whose action is simply $S_{0}[30]$. This result does not depend on $S_{0}$ being a free-field action but merely on its Galilei-invariance [3]. In order to study the properties of the stochastic Langevin equation (1.1), it is hence sufficient

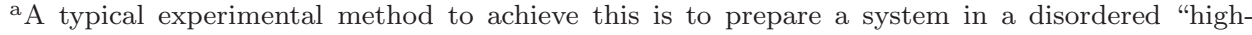
temperature" initial state and then to "quench" it by lowering very rapidly the temperature below the critical temperature $T_{\mathrm{C}}$ such that ergodicity is broken and several distinct steady-states appear.
} 


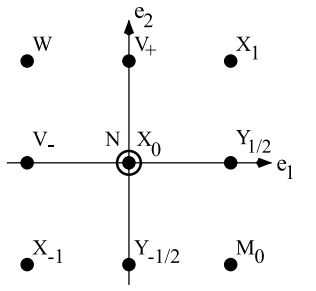

(a)

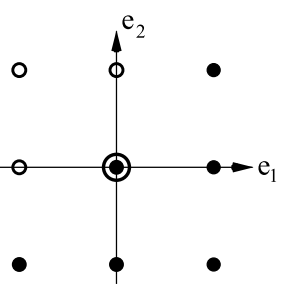

(b)

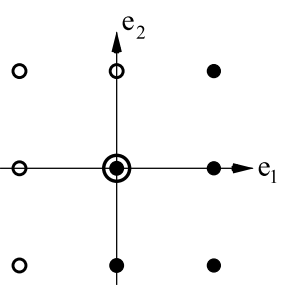

(c)

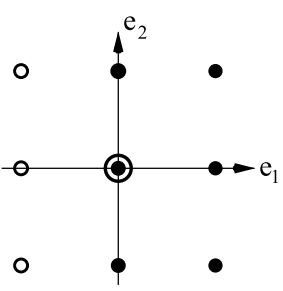

(d)

Fig. 1. (a) Root diagram of the complex Lie algebra $B_{2}$ and the identification of the generators (1.4) of the complexified conformal Lie algebra $\left(\mathfrak{c o n f}_{3}\right)_{\mathbb{C}} \supset(\mathfrak{s c h})_{1}$. The double circle in the center denotes the Cartan subalgebra. The generators belonging to the three non-isomorphic parabolic subalgebras $[20]$ are indicated by the full points, namely (b) $\widetilde{\mathfrak{s c h}}_{1}$, (c) $\widetilde{\mathfrak{a g e}}_{1}$ and (d) $\widetilde{\mathfrak{a l t}}_{1}$.

to concentrate on the properties of its deterministic part (where $\eta$ is dropped) which reduces the problem to the study of those dynamical symmetries of nonlinear partial differential equations which extend dynamical scaling. For a systematic exposition, with many explicit tests, see [24]. The symmetry properties of the deterministic part of this kind of problems will be studied in this paper.

In the context of phase-ordering kinetics, the Schrödinger algebra $\mathfrak{s c h}_{1}[29]$ has played an important role. ${ }^{\mathrm{b}}$ In what follows we shall restrict to one space dimension and we recall in Fig. 1 through a root diagram the definition of $\widetilde{\mathfrak{s c h}}_{1}$ as a parabolic subalgebra of the conformal algebra $\mathfrak{c o n f}_{3}[6,20]$. The inclusion $\mathfrak{s c h}_{1} \subset\left(\mathfrak{c o n f}_{3}\right)_{\mathbb{C}}$ can be realized by considering the "mass" $\mathcal{M}$ as an additional coordinate. It is convenient to perform a Fourier-Laplace transform with respect to $\mathcal{M}$, with the dual coordinate $\zeta$. Then the generators of $\left.(\mathfrak{c o n f})_{3}\right)_{\mathbb{C}}$ read explicitly

$$
\begin{aligned}
X_{-1} & =-\partial_{t}, \quad Y_{-\frac{1}{2}}=-\partial_{r}, \quad M_{0}=\mathrm{i} \partial_{\zeta}, \quad Y_{\frac{1}{2}}=-t \partial_{r}+\mathrm{i} r \partial_{\zeta}, \\
X_{0} & =-t \partial_{t}-\frac{1}{2} r \partial_{r}-\frac{x}{2}, \quad X_{1}=-t^{2} \partial_{t}-t r \partial_{r}+\frac{\mathrm{i}}{2} r^{2} \partial_{\zeta}-x t, \\
N & =-t \partial_{t}+\zeta \partial_{\zeta}, \quad W=-\zeta^{2} \partial_{\zeta}-\zeta r \partial_{r}-\frac{1}{2} r^{2} \partial_{t}-x \zeta, \\
V_{-} & =-\zeta \partial_{r}-r \partial_{t}, \quad V_{+}=-2 t r \partial_{t}-2 \zeta r \partial_{\zeta}-\left(r^{2}+2 \mathrm{i} \zeta t\right) \partial_{r}-2 x r,
\end{aligned}
$$

and the correspondence with the root vectors is illustrated in Fig. 1(a).

The complete list of non-isomorphic parabolic subalgebras of $\left(\mathfrak{c o n f}_{3}\right)_{\mathbb{C}}$ is as follows [20]:

$$
\begin{aligned}
\widetilde{\mathfrak{s c h}_{1}} & =\left\langle X_{-1,0,1}, Y_{-\frac{1}{2}, \frac{1}{2}}, M_{0}, N\right\rangle, \\
\widetilde{\mathfrak{a g e}}_{1} & =\left\langle X_{0,1}, Y_{-\frac{1}{2}, \frac{1}{2}}, M_{0}, N\right\rangle, \\
\widetilde{\mathfrak{a l t}} & =\left\langle D, X_{1}, Y_{-\frac{1}{2}, \frac{1}{2}}, M_{0}, N, V_{+}\right\rangle
\end{aligned}
$$

\footnotetext{
${ }^{\mathrm{b}}$ While in the literature it is usually stated that the Lie algebra $\mathfrak{s c h} \mathfrak{h}_{1}$ was first written down by Lie [27], its elements already occur almost 40 years earlier in Jacobi's lectures on analytical mechanics $[26]$.
} 
and these definitions are illustrated in Fig. 1(b)-(d). Here we used the generator $D$ of the full dilatations ${ }^{\mathrm{c}}$

$$
D:=2 X_{0}-N=-t \partial_{t}-r \partial_{r}-\zeta \partial_{\zeta}-x
$$

For applications to non-equilibrium physics, it is of interest to consider as well the corresponding "almost-parabolic subalgebras" without the generator $N$ [32], namely

$$
\begin{aligned}
\mathfrak{s c h}_{1} & =\left\langle X_{-1,0,1}, Y_{ \pm \frac{1}{2}}, M_{0}\right\rangle, \\
\mathfrak{a g e}_{1} & =\left\langle X_{0,1}, Y_{ \pm \frac{1}{2}}, M_{0}\right\rangle, \\
\mathfrak{a l t}_{1} & =\left\langle D, X_{1}, Y_{ \pm \frac{1}{2}}, M_{0}, V_{+}\right\rangle .
\end{aligned}
$$

We shall show in Sec. 2.1 that $\mathfrak{a l t}_{1}$ is isomorphic to the Poincaré algebra $\mathfrak{p}_{3}$ (wellknown from relativistic field-theory) through a non-conventional realization of the latter; a correspondence which at first thought might appear surprising.

While the explicit representation (1.4) concerns the linear free Schrödinger equation $\mathcal{S} \phi=0$, with $\mathcal{S}=2 M_{0} X_{-1}-Y_{-\frac{1}{2}}^{2}$, Schrödinger-invariance can also be proven for semilinear Schrödinger equations of the form

$$
\mathcal{S} \phi=g F(\phi, \widetilde{\phi})
$$

where $g$ is a dimensionful coupling constant, hence it transforms under the action of scaling or conformal transformations. The corresponding representations have been explicitly derived in the case of a variable mass for $\left(\mathfrak{c o n f}_{3}\right)_{\mathbb{C}}[32]$ and its subalgebras and for a fixed mass for $\mathfrak{s c h}_{1}$ and for $\mathfrak{a g e}_{1}[3]$, from which the form of the potential $F$ in (1.8) can be deduced. Supersymmetric extensions of the Schrödinger algebra are discussed in [21].

While these examples and others already illustrate the intensive study of the Schrödinger algebra and of its subalgebras [4], the other nontrivial subalgebra $\mathfrak{a l t}_{1}$ has so far received much less attention. ${ }^{\mathrm{d}}$ One of the few results established so far concerns the non-relativistic limit of the conformal algebra $\left(\mathfrak{c o n f} f_{3}\right)_{\mathbb{C}}$. For a dynamical mass, that is the dynamical symmetry algebra of the massive Klein-Gordon equation

$$
\left(\frac{1}{c^{2}} \frac{\partial^{2}}{\partial t^{2}}+\frac{\partial}{\partial \mathbf{r}} \cdot \frac{\partial}{\partial \mathbf{r}}-\mathcal{M}^{2} c^{2}\right) \phi_{\mathcal{M}}(t, \mathbf{r})=0
$$

and the non-relativistic limit is obtained by letting the speed of light $c \rightarrow \infty$. Contrary to widely held beliefs (which go back at least to [2]), it turned out that

\footnotetext{
${ }^{\mathrm{c}}$ In physics, if one considers the spacetime rescaling $t \mapsto \lambda^{z} t, \mathbf{r} \mapsto \lambda \mathbf{r}$ with constant $\lambda$, the quantity $z$ is called dynamical exponent. Integrating the infinitesimal dilatation generator $X_{0}$ in (1.4), one finds $z=2$, whereas the dilatation generator $D$ in (1.6) gives $z=1$.

dThis algebra had been identified first in [17], under the name of "conformal Galilean algebra" CGA(1). In recent years, especially string theorists have pursued the study of its representations, often in the context of variants of the AdS/CFT correspondence. It can be shown that $\mathfrak{s c h}_{1}$ and $\mathfrak{a l t}_{1}$ are essentially the only possible distinct non-relativistic limits of the conformal algebra, for light-like and time-like geodesics, respectively [10].
} 
in this limit $\left.(\mathfrak{c o n f})_{3}\right)_{\mathbb{C}} \rightarrow \mathfrak{a l t}_{1} \neq \mathfrak{s c h}_{1}$ and furthermore this is not a group contraction [20]. In this paper, we study the Lie structure of $\mathfrak{a l t}_{1}$, give matrix as well as dual representations, characterize Appell systems in connection with coherent states and Leibniz function. The organization of the paper is as follows: Sec. 2 concerns the Lie structure of $\mathfrak{a l t}_{1}$ where in particular we study infinite-dimensional extensions and also discuss applications to the computation of covariant two-point functions. Casimir operators and matrix representations are provided in Sec. 3. Section 4 focuses on Cartan decomposition and dual representations. A smooth introduction to Wick products and Appell polynomials is given in Sec. 5. Appell systems of $\mathfrak{a l t}_{1}$ are characterized in Sec. 6. Calculations concerning coherent states and Leibniz function are contained in Sec. 7 and we conclude in Sec. 8. Some of our results were announced earlier [22].

\section{A Brief Perspective on the Algebra $\mathfrak{a l t}_{1}$}

We shall take in this section a closer look at the abstract Lie algebra $\mathfrak{a l t}_{1}$ and its representations; we shall also see that, like the algebra $\mathfrak{s c h}_{1}$, it can be embedded naturally in an infinite-dimensional Lie algebra $\mathcal{W}$ which is an extension of the algebra $\operatorname{Vect}\left(S^{1}\right)$ of vector fields on the circle. Quite strikingly, we shall find on our way a "no-go theorem" that proves the impossibility of a conventional extension of the embedding $\mathfrak{a l t}_{1} \subset \mathfrak{c o n f}_{3}$ on the one hand, and a surprisingly simple geometric interpretation of $\mathcal{W}$ that hints at a possible connection with $\mathfrak{s v}$.

\subsection{The abstract Lie algebra $\mathfrak{a l t}_{1}$}

Elementary computations make it clear that

$$
\mathfrak{a l t}_{1}=\left\langle V_{+}, D, Y_{-\frac{1}{2}}\right\rangle \ltimes\left\langle X_{1}, Y_{\frac{1}{2}}, M_{0}\right\rangle=: \mathfrak{g} \ltimes \mathfrak{h},
$$

is a semi-direct product of $\mathfrak{g} \cong \mathfrak{s l}(2, \mathbb{R})$ by a three-dimensional commutative Lie algebra $\mathfrak{h}$; the vector space $\mathfrak{h}$ is the irreducible spin- 1 real representation of $\mathfrak{s l}(2, \mathbb{R})$, which can be identified with $\mathfrak{s l}(2, \mathbb{R})$ itself with the adjoint action. So one has the following

Proposition 2.1. The following Lie algebra isomorphisms hold true. First,

$$
\mathfrak{a} \mathfrak{l t}_{1} \cong \mathfrak{s l}(2, \mathbb{R}) \otimes \frac{\mathbb{R}[\varepsilon]}{\varepsilon^{2}},
$$

where $\varepsilon$ is a "Grassmann" variable. Second,

$$
\mathfrak{a l t} \mathfrak{t}_{1} \cong \mathfrak{p}_{3}
$$

where $\mathfrak{p}_{3} \cong \mathfrak{s o}(2,1) \ltimes \mathbb{R}^{3}$ is the relativistic Poincaré algebra in $(2+1)$ dimensions.

Proof. We shall establish the first isomorphism explicitly. Take a basis $\left(L_{1}, L_{0}, L_{-1}\right)$ of $\mathfrak{s l}(2, \mathbb{R})$ such that

$$
\left[L_{0}, L_{1}\right]=-L_{1}, \quad\left[L_{0}, L_{-1}\right]=L_{-1}, \quad\left[L_{1}, L_{-1}\right]=2 L_{0}
$$


These generators may be written in terms of the anticommuting Pauli matrices $\left(\sigma_{x}, \sigma_{y}, \sigma_{z}\right)$ as follows $L_{1}=\left(\sigma_{x}+\mathrm{i} \sigma_{y}\right) / 2, L_{0}=-\sigma_{z} / 2$ and $L_{-1}=-\left(\sigma_{x}-\mathrm{i} \sigma_{y}\right) / 2$. Then let

$$
L_{i}^{\varepsilon}:=L_{i} \otimes \varepsilon
$$

$(i=-1,0,1)$ where $\varepsilon$ is a Grassmann variable. Then the linear map $\Phi: \mathfrak{a l t}_{1} \rightarrow$ $\mathfrak{s l}(2, \mathbb{R}) \otimes \mathbb{R}[\varepsilon] / \varepsilon^{2}$ defined by

$$
\begin{aligned}
& \Phi\left(V_{+}\right)=L_{1}, \quad \Phi(D)=L_{0}, \quad \Phi\left(Y_{-\frac{1}{2}}\right)=L_{-1}, \\
& \Phi\left(X_{1}\right)=\frac{1}{2} L_{1}^{\varepsilon}, \quad \Phi\left(Y_{\frac{1}{2}}\right)=L_{0}^{\varepsilon}, \quad \Phi\left(M_{0}\right)=L_{-1}^{\varepsilon}
\end{aligned}
$$

is a Lie isomorphism.

The second relation is obvious from the Lie isomorphism $\mathfrak{s o}(2,1) \cong \mathfrak{s l}(2, \mathbb{R})$.

In particular, the representations of $\mathfrak{a l t}_{1} \cong \mathfrak{p}_{3}$ are well-known since Wigner studied them in the '30s.

\subsection{Central extensions: an introduction}

Consider any Lie algebra $\mathfrak{g}$ and an antisymmetric real two-form $\alpha$ on $\mathfrak{g}$. Suppose that its Lie bracket [, ] can be "deformed" into a new Lie bracket [, ] on $\tilde{\mathfrak{g}}:=\mathfrak{g} \times \mathbb{R} K$, where $[K, \mathfrak{g}]=0$, by putting $[(X \widehat{, 0),(Y, 0)}]=([X, Y], \alpha(X, Y))$. Then $\tilde{\mathfrak{g}}$ is called a central extension of $\mathfrak{g}$. The Jacobi identity is equivalent with the nullity of the totally antisymmetric three-form $\mathrm{d} \alpha: \Lambda^{3}(\mathfrak{g}) \rightarrow \mathbb{R}$ defined by

$$
\mathrm{d} \alpha(X, Y, Z)=\alpha([X, Y], Z)+\alpha([Y, Z], X)+\alpha([Z, X], Y) .
$$

Now we say that two central extensions $\mathfrak{g}_{1}, \mathfrak{g}_{2}$ of $\mathfrak{g}$ defined by $\alpha_{1}, \alpha_{2}$ are equivalent if $\mathfrak{g}_{2}$ can be obtained from $\mathfrak{g}_{1}$ by substituting $(X, c) \mapsto(X, c+\lambda(X))(X \in \mathfrak{g})$ for a certain one-form $\lambda \in \mathfrak{g}^{*}$, that is, by changing the non-intrinsic embedding of $\mathfrak{g}$ into $\tilde{\mathfrak{g}}_{1}$. In other words, $\alpha_{1}$ and $\alpha_{2}$ are equivalent if $\alpha_{2}-\alpha_{1}=\mathrm{d} \lambda$, where $\mathrm{d} \lambda(X, Y)=\langle\lambda,[X, Y]\rangle$. The operator $\mathrm{d}$ can be made into the differential of a complex (called Chevalley-Eilenberg complex), and the preceding considerations make it clear that the classes of equivalence of central extensions of $\mathfrak{g}$ make up a vector space $H^{2}(\mathfrak{g})=Z^{2}(\mathfrak{g}) / B^{2}(\mathfrak{g})$, where $Z^{2}$ is the space of cocycles $\alpha \in \Lambda^{2}\left(\mathfrak{g}^{*}\right)$ verifying $\mathrm{d} \alpha=0$, and $B^{2}$ is the space of coboundaries $\mathrm{d} \lambda, \lambda \in \mathfrak{g}^{*}$.

Let us see how this applies to $\mathfrak{a l t}_{1}$.

Proposition 2.2. The Lie algebra $\mathfrak{a l t}_{1}$ has no nontrivial central extension: $H^{2}\left(\mathfrak{a l t}_{1}\right)=0$.

Proof. Of course, this is a consequence of the fact that Poincaré algebras have no nontrivial central extensions, but let us give a proof in this simple example to see how computations work. Note that ad $L_{0}$ acts diagonally on the generators 
$\left(L_{i}\right)$ and $\left(L_{i}^{\varepsilon}\right)(i=-1,0,1)$, defining a $(-1,0,1)$-valued graduation $\delta$ on $\mathfrak{a l t}_{1}$. It is then well known that $\alpha$ is cohomologous to a cocycle $\alpha^{\prime}$ such that $\alpha^{\prime}\left(Z_{i}, Z_{j}\right)=0$ if $Z_{i}, Z_{j}$ are homogeneous generators such that $\delta\left(Z_{i}\right)+\delta\left(Z_{j}\right) \neq 0$ (see for instance [16], Chap. 4), so we may just as well assume this is already the case for $\alpha$. Then $\alpha$ is defined by

$$
\begin{array}{ll}
a:=\alpha\left(L_{1}, L_{-1}\right), & a^{\varepsilon}:=\alpha\left(L_{1}^{\varepsilon}, L_{-1}^{\varepsilon}\right), \\
b:=\alpha\left(L_{1}, L_{-1}^{\varepsilon}\right), & b^{\varepsilon}:=\alpha\left(L_{1}^{\varepsilon}, L_{-1}\right), \quad c:=\alpha\left(L_{0}, L_{0}^{\varepsilon}\right) .
\end{array}
$$

The nontrivial Jacobi identities

$$
\begin{aligned}
& 0=\mathrm{d} \alpha\left(L_{1}, L_{-1}, L_{0}^{\varepsilon}\right)=2 c+b-b^{\varepsilon}, \quad 0=\mathrm{d} \alpha\left(L_{1}^{\varepsilon}, L_{-1}, L_{0}^{\varepsilon}\right)=a^{\varepsilon}, \\
& 0=\mathrm{d} \alpha\left(L_{1}, L_{-1}^{\varepsilon}, L_{0}^{\varepsilon}\right)=a^{\varepsilon}, \quad 0=\mathrm{d} \alpha\left(L_{1}^{\varepsilon}, L_{-1}, L_{0}\right)=-2 c
\end{aligned}
$$

give $c=a^{\varepsilon}=0$ and $b=b^{\varepsilon}$. But the central extension $\alpha$ is then trivial: it is killed by substituting $L_{0} \rightarrow L_{0}+2 a K, L_{0}^{\varepsilon} \rightarrow L_{0}^{\varepsilon}+2 b K$.

A new situation arises upon embedding $\mathfrak{a l t}_{1}$ into an infinite-dimensional Lie algebra.

Remark 2.1. In $d=2$ spatial dimensions, a so-called 'exotic' central extension exists for the algebra $\mathfrak{a l t}_{2}[28]$.

\subsection{Infinite-dimensional extension of $\mathfrak{a l t}_{1}$}

The Lie algebra $\operatorname{Vect}\left(S^{1}\right)$ of vector fields on the circle has a long story in mathematical physics. It was discovered by Virasoro in $1970[34,8]$ that $\operatorname{Vect}\left(S^{1}\right)$ has a one-parameter family of central extensions which yield the so-called Virasoro algebra

$$
\mathfrak{v i r}:=\operatorname{Vect}\left(S^{1}\right) \oplus \mathbb{R} K=\left\langle\left(L_{n}\right)_{n \in \mathbb{Z}}, K\right\rangle
$$

with Lie brackets ( $c \in \mathbb{R}$ is a parameter and is called the central charge)

$$
\left[K, L_{n}\right]=0, \quad\left[L_{n}, L_{m}\right]=(n-m) L_{n+m}+\delta_{n+m, 0} \frac{c}{12} n\left(n^{2}-1\right) K .
$$

When $c=0$, one retrieves $\operatorname{Vect}\left(S^{1}\right)$ by identifying the $\left(L_{n}\right)$ with the usual Fourier basis $\left(e^{\mathrm{i} n \theta} \mathrm{d} \theta\right)_{n \in \mathbb{Z}}$ of periodic vector fields on $[0,2 \pi]$, or with $L_{n} \mapsto \ell_{n}:=-z^{n+1} \frac{\mathrm{d}}{\mathrm{d} z}$ with $z:=e^{\mathrm{i} \theta}$. Note, in particular, that $\left\langle L_{-1}, L_{0}, L_{1}\right\rangle$ is isomorphic to $\mathfrak{s l}(2, \mathbb{R})$, with Lie brackets given in Sec. 2.1, and that the Virasoro cocycle restricted to $\mathfrak{s l}(2, \mathbb{R})$ is 0 , as should be ( $\operatorname{since} \mathfrak{s l}(2, \mathbb{R})$ has no nontrivial central extensions).

The Schrödinger algebra $\mathfrak{s c h}_{1}$ can be embedded into the infinite-dimensional Lie algebra $\mathfrak{s v}$ (introduced in 1994, [18]) which is spanned by the generators $\left\langle L_{n}, Y_{m}, M_{n}\right\rangle$, with nonvanishing commutators

$$
\begin{aligned}
{\left[L_{n}, L_{p}\right] } & =(n-p) L_{n+p}, \quad\left[L_{n}, Y_{m}\right]=(n / 2-m) Y_{n+m}, \\
{\left[L_{n}, M_{p}\right] } & =-p M_{n+p}, \quad\left[Y_{m}, Y_{m^{\prime}}\right]=\left(m-m^{\prime}\right) M_{m+m^{\prime}}
\end{aligned}
$$

with $n, p \in \mathbb{Z}$ and $m, m^{\prime} \in \mathbb{Z}+\frac{1}{2}$. Note that $\mathfrak{s v}$ is a semi-direct product of $\operatorname{Vect}\left(S^{1}\right)$ with an infinite-dimensional nilpotent Lie algebra. Its mathematical structure is 


\section{Henkel et al.}

analyzed in detail in $[31,33]$ and supersymmetric extensions are discussed in [21]. There is only one class of central extensions of $\mathfrak{s v}$, given by the extension by zero of the Virasoro cocycle [18].

An analogous embedding holds for $\mathfrak{a l t}_{1}$, namely $\mathfrak{a l t} \mathfrak{t}_{1} \cong \mathfrak{s l}(2, \mathbb{R}) \otimes \mathbb{R}[\varepsilon] / \varepsilon^{2}$ can be embedded into the Lie algebra

$$
\mathcal{W}:=\operatorname{Vect}\left(S^{1}\right) \otimes \frac{\mathbb{R}[\varepsilon]}{\varepsilon^{2}}=\left\langle L_{n}\right\rangle_{n \in \mathbb{Z}} \ltimes\left\langle L_{n}^{\varepsilon}\right\rangle_{n \in \mathbb{Z}},
$$

with Lie bracket

$$
\left[L_{n}, L_{m}\right]=(n-m) L_{n+m}, \quad\left[L_{n}, L_{m}^{\varepsilon}\right]=(n-m) L_{n+m}^{\varepsilon}, \quad\left[L_{n}^{\varepsilon}, L_{m}^{\varepsilon}\right]=0 .
$$

These brackets come out naturally when one puts $\mathcal{W}$ in the $2 \times 2$-matrix form

$$
L_{n} \mapsto\left(\begin{array}{cc}
\ell_{n} & 0 \\
0 & \ell_{n}
\end{array}\right), L_{n}^{\varepsilon} \mapsto\left(\begin{array}{cc}
0 & \ell_{n} \\
0 & 0
\end{array}\right)
$$

leading to straightforward generalizations (see, in particular, $[31,33]$ for a deformation of $\mathfrak{s} \mathfrak{v}$ that can be represented as upper-triangular $3 \times 3$ Virasoro matrices instead).

In terms of the standard representations of $\operatorname{Vect}\left(S^{1}\right)$ as modules of $\alpha$-densities $\mathcal{F}_{\alpha}=\left\{u(z)(\mathrm{d} z)^{\alpha}\right\}$ with the action

$$
f(z) \frac{\mathrm{d}}{\mathrm{d} z}\left(u(z)(\mathrm{d} z)^{\alpha}\right)=\left(f u^{\prime}+\alpha f^{\prime} u\right)(z)(\mathrm{d} z)^{\alpha},
$$

we have

\section{Proposition 2.3.}

$$
\mathcal{W} \cong \operatorname{Vect}\left(S^{1}\right) \ltimes \mathcal{F}_{-1}
$$

Proof. Immediate from the obvious isomorphism of $\operatorname{Vect}\left(S^{1}\right)$ (with the adjoint action) with the $\operatorname{Vect}\left(S^{1}\right)$-module $\mathcal{F}_{-1}$.

It can be easily shown that $\mathcal{W}$ has two linearly independent central extensions:

- the natural extension to $\mathcal{W}$ of the Virasoro cocycle on $\operatorname{Vect}\left(S^{1}\right)$, namely $\left.[]=,\widetilde{[},\right]$ except for $\left[\widetilde{L_{n}, L_{-n}}\right]=n\left(n^{2}-1\right) K+2 n L_{0}$. In other words, $\operatorname{Vect}\left(S^{1}\right)$ is centrally extended, but its action on $\mathcal{F}_{-1}$ remains unchanged.

- the cocycle $\omega$ which is zero on $\Lambda^{2}\left(\operatorname{Vect}\left(S^{1}\right)\right)$ and $\Lambda^{2}\left(\mathcal{F}_{-1}\right)$, and defined on $\operatorname{Vect}\left(S^{1}\right) \times \mathcal{F}_{-1}$ by

$$
\omega\left(L_{n}, L_{m}^{\varepsilon}\right)=\delta_{n+m, 0} n\left(n^{2}-1\right) K^{\varepsilon} .
$$

The independence of these two central charges is nicely illustrated through the following example: consider the generators $V_{n}$ and $V_{n}^{\prime}(n \in \mathbb{Z})$ of two commuting 
Virasoro algebras with central charges $c$ and $c^{\prime}$. Then identify

$$
\begin{aligned}
L_{n} \mapsto\left(\begin{array}{cc}
V_{n}+V_{n}^{\prime} & 0 \\
0 & V_{n}+V_{n}^{\prime}
\end{array}\right), \quad L_{n}^{\varepsilon} \mapsto\left(\begin{array}{cc}
0 & V_{n} \\
0 & 0
\end{array}\right), \\
K \mapsto\left(\begin{array}{ll}
1 & 0 \\
0 & 1
\end{array}\right), \quad K^{\varepsilon} \mapsto\left(\begin{array}{ll}
0 & 1 \\
0 & 0
\end{array}\right)
\end{aligned}
$$

and the nonvanishing commutators become

$$
\begin{aligned}
& {\left[L_{n}, L_{m}\right]=(n-m) L_{n+m}+\frac{c+c^{\prime}}{12}\left(n^{3}-n\right) \delta_{n+m, 0} K} \\
& {\left[L_{n}, L_{m}^{\varepsilon}\right]=(n-m) L_{n+m}^{\varepsilon}+\frac{c}{12}\left(n^{3}-n\right) \delta_{n+m, 0} K^{\varepsilon}}
\end{aligned}
$$

A natural related question is: can one deform the extension of $\operatorname{Vect}\left(S^{1}\right)$ by the $\operatorname{Vect}\left(S^{1}\right)$-module $\mathcal{F}_{-1}$ ? The answer is: no, thanks to the triviality of the cohomology space $H^{2}\left(\operatorname{Vect}\left(S^{1}\right), \mathcal{F}_{-1}\right)$ (see [15] or [16]). In other words, any Lie algebra structure $[$,$] on the vector space \operatorname{Vect}\left(S^{1}\right) \oplus \mathcal{F}_{-1}$ such that

$[(X, \widehat{\phi),(Y}, \psi)]=\left([X, Y]_{\operatorname{Vect}\left(S^{1}\right)}, \operatorname{ad}_{\operatorname{Vect}\left(S^{1}\right)} X . \psi-\operatorname{ad}_{\operatorname{Vect}\left(S^{1}\right)} Y . \phi+B(X, Y)\right)$

( $B$ antisymmetric two-form on $\operatorname{Vect}\left(S^{1}\right)$ ) is isomorphic to the Lie structure of $\mathcal{W}$.

So one may say that $\mathcal{W}$ and its central extensions are natural objects to look at.

\subsection{Some results on representations of $\mathcal{W}$}

We shall give in this subsection several results, the second of which certainly deserves deeper thoughts and will be developed in the future.

Proposition 2.4. ("no-go theorem") There is no way to extend the usual representation of $\mathfrak{a l t}_{1}$ as conformal vector fields into an embedding of $\mathcal{W}$ into the Lie algebra of vector fields on $\mathbb{R}^{3}$.

Proof. Put $L_{2}^{\varepsilon}=f \partial_{t}+g \partial_{r}+h \partial_{\zeta}$ where $f=f(t, r, \zeta), g=g(t, r, \zeta), h=h(t, r, \zeta)$ are yet undetermined functions. We use the explicit forms of the generators of $\mathfrak{a l t}_{1}$. Then the relations

$$
\left[L_{2}^{\varepsilon}, L_{-1}\right]=3 L_{1}^{\varepsilon}, \quad\left[L_{2}^{\varepsilon}, L_{-1}^{\varepsilon}\right]=0, \quad\left[L_{2}^{\varepsilon}, L_{0}^{\varepsilon}\right]=0
$$

give respectively

$$
\begin{aligned}
& \partial_{r} f=-6 t^{2}, \quad \partial_{r} g=-6 t r, \quad \partial_{r} h=3 \mathrm{i} r^{2}, \\
& \partial_{\zeta} f=\partial_{\zeta} g=\partial_{\zeta} h=0, \\
& \partial_{r} f=0, \quad f=t r \partial_{r} g, \quad \mathrm{i} g+t \partial_{r} h=0 .
\end{aligned}
$$

But (2.17) and (2.19) are incompatible.

The following proposition hints at quite unexpected connections between contact structures in $\mathbb{R}^{3}$, the Lie algebra $\mathfrak{s v}$ and the Lie algebra $\mathcal{W}$. Recall that a contact 
form $\alpha$ on a three-dimensional manifold $\mathcal{V}$ is a one-form on $\mathcal{V}$ such that $\mathrm{d} \alpha \wedge \alpha$ is a non-degenerate volume form.

Proposition 2.5. Let $\alpha$ be the complex-valued contact form on $\mathbb{R}^{3}$ defined by $\alpha(t, r, \zeta)=r \mathrm{~d} r-2 \mathrm{i} \zeta \mathrm{d} t$. Then the Lie algebra of vector fields $X(t, r, \zeta)$ such that:

(i) $\mathcal{L}_{X} \alpha$ is proportional to $\alpha$, i.e. $\mathcal{L}_{X} \alpha=f \alpha$ for a certain function $f=f(t, r, \zeta)$;

(ii) $\left[X, \partial_{\zeta}\right]=0$, i.e. components of $X$ do not depend on $\zeta$;

(iii) $\mathcal{L}_{X} \mathrm{~d} t$ is proportional to $\mathrm{d} t$, so that $X$ is tangent to each leaf $t=$ constant is generated by $L_{n}, L_{n}^{\varepsilon}, n \in \mathbb{Z}$, with

$$
\begin{aligned}
& L_{n}=-t^{n+1} \partial_{t}-\frac{1}{2}(n+1) t^{n} r \partial_{r}+\frac{\mathrm{i}}{4}(n+1) n t^{n-1} r^{2} \partial_{\zeta}-\frac{x}{2}(n+1) t^{n}, \\
& L_{n}^{\varepsilon}=-t^{n+1} \frac{1}{r} \partial_{r}+\frac{\mathrm{i}}{2}(n+1) t^{n} \partial_{\zeta} .
\end{aligned}
$$

The Lie algebra $\left\langle L_{n}, L_{n}^{\varepsilon}\right\rangle$ is isomorphic to $\mathcal{W}$, with commutators given by formula (2.9) in Sec. 2.3.

The attentive reader will have noted that $L_{n}=X_{n}$ and $L_{n}^{\varepsilon}=\frac{1}{r} Y_{n+\frac{1}{2}}[20]$.

Proposition 2.6. The infinite-dimensional extension $\mathcal{W}$ of the algebra $\mathfrak{a l t}_{1}$ is a contraction of a pair of commuting loop algebras $\operatorname{Vect}\left(S^{1}\right) \oplus \overline{\operatorname{Vect}\left(S^{1}\right)} \rightarrow \mathcal{W}$. In particular, we have the explicit differential operator representation

$$
\begin{aligned}
& L_{n}=-t^{n+1} \partial_{t}-(n+1) t^{n} r \partial_{r}-(n+1) x t^{n}-n(n+1) \gamma t^{n-1} r, \\
& L_{n}^{\varepsilon}=-t^{n+1} \partial_{r}-(n+1) \gamma t^{n},
\end{aligned}
$$

where $x$ and $\gamma$ are parameters and $n \in \mathbb{Z}$.

Proof. Let $\ell_{n}$ and $\bar{\ell}_{n}$ be the generators of the two commuting loop algebras $\operatorname{Vect}\left(S^{1}\right)$ and $\overline{\operatorname{Vect}\left(S^{1}\right)}$. Obviously, the generators $X_{n}:=\ell_{n}+\bar{\ell}_{n}$ and $Y_{n}:=a \bar{\ell}_{n}$ satisfy the commutation relations

$$
\left[X_{n}, X_{m}\right]=(n-m) X_{n+m},\left[X_{n}, Y_{m}\right]=(n-m) Y_{n+m},\left[Y_{n}, Y_{m}\right]=a(n-m) Y_{n+m}
$$

which in the limit $a \rightarrow 0$ reduces to (2.9). A differential-operator representation of the $X_{n}$ and $Y_{n}$ is given in case (iii) of Table 1 of [19] and $L_{n}=\lim _{a \rightarrow 0} X_{n}$ and $L_{n}^{\varepsilon}=\lim _{a \rightarrow 0} Y_{n}$ which yields the form $(2.21)$.

One of the possible applications of these generators is the calculation of multipoint correlation functions of many-body systems. One says that the $n$-point correlator $F_{n}:=\left\langle\Phi_{1} \cdots \Phi_{n}\right\rangle$ of so-called quasi-primary fields $\Phi_{j}$ is covariant under the action of the generators $\left(\mathcal{X}_{i}\right)_{i \in I}$ of a Lie algebra if $\mathcal{X}_{i} F_{n}=0$ for all $i \in I$. We apply this idea to the two-point correlators covariant under $\mathfrak{a l t}_{1}$. The standard representation (1.4) refers to the coordinates $\zeta, t, r$ and the quasi-primary field will 
be denoted by $\psi=\psi(\zeta, t, r)$ and is assumed to have a scaling dimension $x$. The two-point function reads [20]

$$
\begin{aligned}
\left\langle\psi_{1}\left(\zeta_{1}, t_{1}, r_{1}\right) \psi_{2}\left(\zeta_{2}, t_{2}, r_{2}\right)\right\rangle= & \left(t_{1}-t_{2}\right)^{-\left(x_{1}+x_{2}\right) / 2}\left(\frac{t_{1}}{t_{2}}\right)^{\left(x_{2}-x_{1}\right) / 2} \\
& \times f\left(\zeta_{1}-\zeta_{2}+\frac{\mathrm{i}}{2} \frac{\left(r_{1}-r_{2}\right)^{2}}{t_{1}-t_{2}}\right),
\end{aligned}
$$

where $f$ is an arbitrary function. Similarly, imposing covariance under the representation (2.20) leads to

$$
\left\langle\psi_{1}\left(\zeta_{1}, t_{1}, r_{1}\right) \psi_{2}\left(\zeta_{2}, t_{2}, r_{2}\right)\right\rangle=\delta_{x_{1}, x_{2}}\left(t_{1}-t_{2}\right)^{-x_{1}} f\left(\zeta_{1}+\zeta_{2}+\frac{\mathrm{i}}{2} \frac{r_{1}^{2}-r_{2}^{2}}{t_{1}-t_{2}}\right),
$$

where again $f$ is an arbitrary function. It is evident that these two representations will describe quite distinct physical systems.

Furthermore, instead to working with the variable $\zeta$, it is from a physical point of view more natural to consider the Fourier-transform of the field $\psi$ with respect to $\zeta$ and to define $[20]$

$$
\phi(t, r)=\phi_{\mathcal{M}}(t, r):=\frac{1}{\sqrt{2 \pi}} \int_{\mathbb{R}} \mathrm{d} \zeta e^{-\mathrm{i} \mathcal{M} \zeta} \psi(\zeta, t, r) .
$$

Then the quasiprimary fields $\phi$ are characterized by $\mathcal{M}$ and their scaling dimension. We find the following two-point correlation functions

- for the standard representation we find from (2.22) [20]

$$
\begin{aligned}
\left\langle\phi_{1}\left(t_{1}, r_{1}\right) \phi_{2}^{*}\left(t_{2}, r_{2}\right)\right\rangle= & \phi_{0} \delta\left(\mathcal{M}_{1}-\mathcal{M}_{2}\right) \Theta\left(t_{1}-t_{2}\right)\left(\frac{t_{1}}{t_{2}}\right)^{\left(x_{2}-x_{1}\right) / 2} \\
& \times\left(t_{1}-t_{2}\right)^{-\left(x_{1}+x_{2}\right) / 2} \exp \left[-\frac{\mathcal{M}_{1}}{2} \frac{\left(r_{1}-r_{2}\right)^{2}}{t_{1}-t_{2}}\right],
\end{aligned}
$$

where $\Theta(t)$ is the Heaviside function and $\phi_{0}$ a normalization constant.

- for the representation (2.20), we find from (2.23)

$$
\begin{aligned}
\left\langle\phi_{1}\left(t_{1}, r_{1}\right) \phi_{2}\left(t_{2}, r_{2}\right)\right\rangle= & \phi_{0} \delta_{x_{1}, x_{2}} \delta\left(\mathcal{M}_{1}-\mathcal{M}_{2}\right)\left(t_{1}-t_{2}\right)^{-x_{1}} \\
& \times \exp \left[-\frac{\mathcal{M}_{1}}{2} \frac{r_{1}^{2}-r_{2}^{2}}{t_{1}-t_{2}}\right]
\end{aligned}
$$

- finally, for the representation (2.21), the quasiprimary field $\phi(t, r)$ is characterized by its scaling dimension $x$ and the extra parameter $\gamma$. One has [19]

$$
\left\langle\phi_{1}\left(t_{1}, r_{1}\right) \phi_{2}\left(t_{2}, r_{2}\right)\right\rangle=\phi_{0} \delta_{x_{1}, x_{2}} \delta_{\gamma_{1}, \gamma_{2}}\left(t_{1}-t_{2}\right)^{-x_{1}} \exp \left[-2 \gamma_{1} \frac{r_{1}-r_{2}}{t_{1}-t_{2}}\right] .
$$

Clearly, the form of these two-point correlators, notably their invariance under timeor space-translations, are different. 


\section{Casimir Operators and Matrix Representations for $\mathfrak{a l t}_{1}$}

From now on, we consider the finite-dimensional representations of $\mathfrak{a l t}_{1}$. We shall use the following notational conventions (see Sec. 2.1):

- $\mathfrak{s l}(2, \mathbb{R})$ is spanned by $X_{ \pm 1}, X_{0}$.

- The commutative algebra $h$ is spanned by $Y_{ \pm 1}, Y_{0}$.

The nonzero commutators of $\mathfrak{a l t}_{1}$ are:

$$
\left[X_{n}, X_{m}\right]=(n-m) X_{n+m}, \quad\left[X_{n}, Y_{m}\right]=(n-m) Y_{n+m} ; \quad n, m \in\{ \pm 1,0\} .
$$

In the notation of Sec. $2, X_{n}=L_{n}$ and $Y_{n}=L_{n}^{\varepsilon}$.

\subsection{Casimir operators}

The construction of such operators is important, because in the vector-field representation they correspond to the invariant differential operators. Looking for second-order differential operator we write:

$$
\hat{S}=a_{i j} X_{i} X_{j}+b_{i j} X_{i} Y_{j}+c_{i j} Y_{i} Y_{j}+d_{i} X_{i}+e_{i} Y_{i}
$$

Here the sum over repeated index is understood and $i, j \in\{ \pm 1,0\}$. From the conditions $\left[\hat{S}, X_{i}\right]=\left[\hat{S}, Y_{i}\right]=0$ the coefficients $a, b, c, d, e$ can be determined. The result is the following:

$$
\begin{aligned}
\hat{S} & =A S_{0}+S_{1}, \quad A=\text { cste } \\
S_{0} & =X_{-1} Y_{1}+X_{1} Y_{-1}-2 X_{0} Y_{0} \\
S_{1} & =Y_{-1} Y_{1}-Y_{0}^{2} .
\end{aligned}
$$

The calculation for different representations gives the results:

- In the physical representation (1.4)

$$
\hat{S}=-t^{2}\left(2 \mathrm{i} \partial_{\zeta} \partial_{t}+\partial_{r}^{2}\right)-\mathrm{i} t(2 x-1) \partial_{\zeta}
$$

which for the canonical scaling dimension of the wave function $x=\frac{1}{2}$ reduces to the usual Schrödinger-operator in dynamical-mass representation, see [20, 32].

- In the representation $(2.20)$

$$
\hat{S}=\mathrm{i} A\left(\frac{x}{2}-1\right) \partial_{\zeta}-\frac{1}{4} \partial_{\zeta}^{2}
$$

The inverse Fourier transformation with respect to $\zeta$ of the wave function leads to a constant.

- Finally for the representation (2.21) this operator is again constant. 
This confirms the statement that the "fixed mass" or projective representation of $\mathfrak{a l t}_{1}$ characterizes the wave function with its scaling dimension $x$ and the constant $\gamma$ instead of mass (the mass generator drops out). ${ }^{\mathrm{e}}$

Remark 3.1. In fact, when consider $d$ spacial dimensions that is a representation of $\mathfrak{a l t}_{d}$, the compatibility with rotations require this constant $\gamma$ to be a vector [7]

$$
\gamma \rightarrow \gamma=\left(\gamma_{1}, \ldots, \gamma_{d}\right)
$$

\subsection{Matrix representations}

The adjoint representation can be obtained directly from the commutators $\left(\left[\eta_{i}, \eta_{j}\right]=c_{i j}^{k} \eta_{k}\right)$ in the following $6 \times 6$-matrix form

$$
Y_{-1}=c_{1 j}^{k}=\left(\begin{array}{rrrrrr}
0 & 0 & 0 & 0 & -1 & 0 \\
0 & 0 & 0 & 0 & 0 & -2 \\
0 & 0 & 0 & 0 & 0 & 0 \\
0 & 0 & 0 & 0 & 0 & 0 \\
0 & 0 & 0 & 0 & 0 & 0 \\
0 & 0 & 0 & 0 & 0 & 0
\end{array}\right), \quad Y_{0}=c_{2 j}^{k}=\left(\begin{array}{rrrrrr}
0 & 0 & 0 & 1 & 0 & 0 \\
0 & 0 & 0 & 0 & 0 & 0 \\
0 & 0 & 0 & 0 & 0 & -1 \\
0 & 0 & 0 & 0 & 0 & 0 \\
0 & 0 & 0 & 0 & 0 & 0 \\
0 & 0 & 0 & 0 & 0 & 0
\end{array}\right) \text {, }
$$

$$
Y_{1}=c_{3 j}^{k}=\left(\begin{array}{rrrrrr}
0 & 0 & 0 & 0 & 0 & 0 \\
0 & 0 & 0 & 0 & 2 & 0 \\
0 & 0 & 0 & 0 & 0 & 1 \\
0 & 0 & 0 & 0 & 0 & 0 \\
0 & 0 & 0 & 0 & 0 & 0 \\
0 & 0 & 0 & 0 & 0 & 0
\end{array}\right), \quad X_{-1}=c_{4 j}^{k}=\left(\begin{array}{rrrrrr}
0 & 0 & 0 & 0 & 0 & 0 \\
0 & -1 & 0 & 0 & 0 & 0 \\
0 & 0 & -2 & 0 & 0 & 0 \\
0 & 0 & 0 & 0 & 0 & 0 \\
0 & 0 & 0 & 0 & -1 & 0 \\
0 & 0 & 0 & 0 & 0 & -2
\end{array}\right),
$$

$$
X_{0}=c_{5 j}^{k}=\left(\begin{array}{rrrrrr}
1 & 0 & 0 & 0 & 0 & 0 \\
0 & 0 & 0 & 0 & 0 & 0 \\
0 & 0 & -1 & 0 & 0 & 0 \\
0 & 0 & 0 & 1 & 0 & 0 \\
0 & 0 & 0 & 0 & 0 & 0 \\
0 & 0 & 0 & 0 & 0 & -1
\end{array}\right), \quad X_{1}=c_{6 j}^{k}=\left(\begin{array}{rrrrrr}
0 & 2 & 0 & 0 & 0 & 0 \\
0 & 0 & 1 & 0 & 0 & 0 \\
0 & 0 & 0 & 0 & 0 & 0 \\
0 & 0 & 0 & 0 & 2 & 0 \\
0 & 0 & 0 & 0 & 0 & 1 \\
0 & 0 & 0 & 0 & 0 & 0
\end{array}\right)
$$

e If one admits values $z \neq 1,2$ for the dynamical exponent, there are no representations of $\mathfrak{a l t}_{1}$ in terms of local differential operators. However, for generic values of $z$, nonlocal representations in terms for fractional differential operators can be constructed, which can be shown to close on the solution space of an appropriate linear PDE of fractional order [25]. 
An embedding into $\mathfrak{s u}(4)$ can be obtained by taking the six-dimensional restriction of the algebra $\mathcal{W}$ and writing the generators in its Pauli-matrix form

$$
\begin{gathered}
Y_{-1}=\left(\begin{array}{rrrr}
0 & 0 & 0 & 0 \\
0 & 0 & -1 & 0 \\
0 & 0 & 0 & 0 \\
0 & 0 & 0 & 0
\end{array}\right), \quad Y_{0}=\frac{1}{2}\left(\begin{array}{rrrr}
0 & 0 & -1 & 0 \\
0 & 0 & 0 & 1 \\
0 & 0 & 0 & 0 \\
0 & 0 & 0 & 0
\end{array}\right), \\
Y_{1}=\left(\begin{array}{llll}
0 & 0 & 0 & 1 \\
0 & 0 & 0 & 0 \\
0 & 0 & 0 & 0 \\
0 & 0 & 0 & 0
\end{array}\right), \quad X_{-1}=\left(\begin{array}{rrrr}
0 & 0 & 0 & 0 \\
-1 & 0 & 0 & 0 \\
0 & 0 & 0 & 0 \\
0 & 0 & -1 & 0
\end{array}\right), \\
X_{0}=\frac{1}{2}\left(\begin{array}{rrrr}
-1 & 0 & 0 & 0 \\
0 & 1 & 0 & 0 \\
0 & 0 & -1 & 0 \\
0 & 0 & 0 & 1
\end{array}\right), \quad X_{1}=\left(\begin{array}{rrrr}
0 & 1 & 0 & 0 \\
0 & 0 & 0 & 0 \\
0 & 0 & 0 & 1 \\
0 & 0 & 0 & 0
\end{array}\right) .
\end{gathered}
$$

\section{Cartan Decomposition and Dual Representations}

It is clear, from the commutation relations, that $\mathfrak{a l t}_{1}$ has the following Cartan decomposition:

$$
\mathfrak{a l t}_{1}=\mathcal{P} \oplus \mathcal{K} \oplus \mathcal{L}=\left\{Y_{1}, X_{1}\right\} \oplus\left\{Y_{0}, X_{0}\right\} \oplus\left\{Y_{-1}, X_{-1}\right\}
$$

and there is a one-to-one correspondence between the subalgebras $\mathcal{P}$ and $\mathcal{L}$.

The typical element $\mathcal{X}$ of the algebra $\mathfrak{a l t}_{1}$ is given by $\mathcal{X}=\sum_{i=1}^{6} \alpha_{i} \eta_{i}$ where $\left\{\eta_{i}\right\}, i=1, \ldots, 6$ is a basis of $\mathfrak{a l t}_{1}$. The $\left\{\alpha_{i}\right\}, i=1, \ldots, 6$ are called coordinates of the first kind. The matrix form is:

$$
\mathcal{X}=\left(\begin{array}{cccc}
-\frac{\alpha_{4}}{2} & \alpha_{2} & -\frac{\alpha_{3}}{2} & \alpha_{1} \\
-\alpha_{6} & \frac{\alpha_{4}}{2} & -\alpha_{5} & \frac{\alpha_{3}}{2} \\
0 & 0 & -\frac{\alpha_{4}}{2} & \alpha_{2} \\
0 & 0 & -\alpha_{6} & -\frac{\alpha_{4}}{2}
\end{array}\right)
$$

The group element (near to identity) can be expressed as:

$$
\exp \left(\alpha_{i} \eta_{i}\right)=g\left(\left\{A_{i}\right\}\right)=\exp \left(A_{1} \eta_{1}\right) \cdots \exp \left(A_{6} \eta_{6}\right)
$$

The $A_{i}, i=1, \ldots, 6$ are called coordinates of the second kind. Here the following correspondence is made $\eta_{1}=Y_{1}, \eta_{2}=X_{1}, \eta_{3}=Y_{0}, \eta_{4}=X_{0}, \eta_{5}=Y_{-1}, \eta_{6}=X_{-1}$. Next, consider the one-parameter subgroup generated by $\mathcal{X}, e^{s \mathcal{X}}$, the coordinates $\alpha$ 
scale by factor $s$, while the coordinates $A$ become functions of the single parameter $s$. Consequently one can write

$$
g(A(s))=e^{s \mathcal{X}} .
$$

Evaluating at $s=1$ gives the coordinate transformation $A=A(\alpha)$, while taking derivatives with respect to $A_{i}$ gives

$$
\mathcal{X} g=\sum_{i} e^{A_{1} \eta_{1}} \cdots e^{A_{i-1} \eta_{i-1}} \dot{A}_{i} \eta_{i} e^{A_{i} \eta_{i}} \cdots e^{A_{6} \eta_{6}}=\dot{A}_{\mu} \partial_{\mu} g
$$

with $\partial_{\mu}=\partial / \partial A_{\mu}$; the dot denotes differentiation with respect to $s$. Further considerations show that the coordinates $A$ contain the complete information about the Lie algebra structure.

We can calculate

$$
g\left(\left\{A_{i}\right\}\right)=e^{-\frac{A_{4}}{2}}\left(\begin{array}{cccc}
1-A_{2} A_{6} e^{A_{4}} & A_{2} e^{A_{4}} & A & \bar{A} \\
-A_{6} e^{A_{4}} & e^{A_{4}} & -\left(A_{5}+\frac{1}{2} A_{3} A_{6}\right) e^{A_{4}} & \frac{1}{2} A_{3} e^{A_{4}} \\
0 & 0 & 1-A_{2} A_{6} e^{A_{4}} & A_{2} e^{A_{4}} \\
0 & 0 & -A_{6} e^{A_{4}} & e^{A_{4}}
\end{array}\right),
$$

where

$$
A=-\left(A_{2} A_{5}+\frac{1}{2} A_{2} A_{3} A_{6}+A_{1} A_{6}\right) e^{A_{4}}-\frac{1}{2} A_{3}, \quad \bar{A}=\left(\frac{1}{2} A_{2} A_{3}+A_{1}\right) e^{A_{4}} .
$$

From (4.6) the second kind coordinates can be given in terms of the elements of the matrix representation of the group (and conversely):

$$
\begin{aligned}
& A_{1}=\frac{g_{14}}{g_{22}}-\frac{g_{12} g_{24}}{\left(g_{22}^{2}\right)}, \quad A_{2}=\frac{g_{12}}{g_{22}}, \quad A_{3}=\frac{2 g_{24}}{g_{22}}, \\
& A_{4}=2 \ln g_{22}, \quad A_{5}=-\frac{g_{23}}{g_{22}}-\frac{g_{24} g_{21}}{\left(g_{22}^{2}\right)}, \quad A_{6}=-\frac{g_{21}}{g_{22}} .
\end{aligned}
$$

The multiplication by basis elements $\eta, g \mapsto g \eta$, acting on the universal enveloping algebra with basis $[n]=\eta^{n}=\eta_{1}^{n_{1}} \cdots \eta_{6}^{n_{6}}$ are realized as left-invariant vector fields $\eta^{*}$, acting on function of $A$ (action commutes with multiplication by group element on the left, so $\eta$ acts on the right), given in terms of pi-matrix $\eta_{i}^{*}=\pi_{i \mu}^{*}(A) \partial_{\mu}$. Similarly, multiplication on the left gives right-invariant vector fields $\eta_{i}^{\ddagger}=\pi_{i \mu}^{\ddagger}(A) \partial_{\mu}$.

The dual representations are defined as realization of the Lie algebra as vector fields in terms of coordinates of the second kind acting on the left or right respectively

$$
\eta_{j} g(A)=\pi_{j \mu}^{\ddagger}(A) \partial_{\mu} g(A), \quad g(A) \eta_{j}=\pi_{j \mu}^{*}(A) \partial_{\mu} g(A) .
$$

The connection between left and right dual representations is given by the following splitting lemma

\section{Lemma 4.1.}

$$
\dot{A}_{k}=\alpha_{\mu} \pi_{\mu k}^{*}(A)=\alpha_{\mu} \pi_{\mu k}^{\ddagger}(A)
$$


with initial values $A_{k}(0)=0, \pi^{*}(0)=\pi^{\ddagger}(0)=I$ is gathered from $[1,12,13]$.

For our case we find

$$
\begin{aligned}
& \pi^{\ddagger}=\left(\begin{array}{cccccc}
1 & 0 & 0 & 0 & 0 & 0 \\
0 & 1 & 0 & 0 & 0 & 0 \\
0 & 0 & 1 & 0 & 0 & 0 \\
-A_{1} & -A_{2} & 0 & 1 & 0 & 0 \\
A_{2}^{2} & 0 & -2 A_{2} & 0 & e^{-A_{4}} & 0 \\
2 A_{1} A_{2} & A_{2}^{2} & -2 A_{1} & -2 A_{2} & -A_{3} e^{-A_{4}} & e^{-A_{4}}
\end{array}\right), \\
& \eta^{\ddagger}=\left(\begin{array}{c}
\partial_{1} \\
\partial_{2} \\
\partial_{3} \\
-A_{1} \partial_{1}-A_{2} \partial_{2}-\partial_{4} \\
A_{2}^{2} \partial_{1}-2 A_{2} \partial_{3}+e^{-A_{4}} \partial_{4} \\
2 A_{1} A_{2} \partial_{1}+A_{2}^{2} \partial_{2}-2 A_{1} \partial_{3}-2 A_{2} \partial_{4}-A_{3} e^{-A_{4}} \partial_{5}-e^{-A_{4}} \partial_{6}
\end{array}\right), \\
& \pi^{*}=\left(\begin{array}{cccccc}
e^{-A_{4}} & 0 & -2 A_{6} & 0 & A_{6}^{2} & 0 \\
-A_{3} e^{-A_{4}} & e^{-A_{4}} & -2 A_{5} & -2 A_{6} & 2 A_{5} A_{6} & A_{6}^{2} \\
0 & 0 & 1 & 0 & -A_{6} & 0 \\
0 & 0 & 0 & 1 & -A_{5} & -A_{6} \\
0 & 0 & 0 & 0 & 1 & 0 \\
0 & 0 & 0 & 0 & 0 & 1
\end{array}\right) \\
& \eta^{*}=\left(\begin{array}{c}
e^{-A_{4}} \partial_{1}-2 A_{6} \partial_{3}+A_{6}^{2} \partial_{5} \\
-A_{3} e^{-A_{4}} \partial_{1}+e^{-A_{4}} \partial_{2}-2 A_{5} \partial_{3}-2 A_{6} \partial_{4}+2 A_{5} A_{6} \partial_{5}+A_{6}^{2} \partial_{6} \\
\partial_{3}-A_{6} \partial_{5} \\
\partial_{4}-A_{5} \partial_{5}-A_{6} \partial_{6} \\
\partial_{5} \\
\partial_{6}
\end{array}\right) .
\end{aligned}
$$

The last representation leads to the physical case (2.21) from [19] if $A_{5} \mapsto$ $-r, A_{6} \mapsto-t$ and one supposes the action of the vector fields on the functions in the form $e^{-\gamma A_{3}} e^{-x A_{4}} f\left(A_{5}, A_{6}\right)$.

\section{Wick Products and Appell Polynomials}

Appell polynomials share many properties with Wick products. In physical literature, the term Wick product is even more popular. The aim of this section is to provide a "smooth" introduction to Appell polynomials through Wick products. The following presentation is gathered from [1]. 
Let $X_{1}, X_{2}, \ldots$ be random variables. The Wick powers are defined inductively on $k$ as follows. Start with $\left\langle X_{1}, X_{2}, \ldots, X_{k}\right\rangle=1$ for $k=0$. Then for any $k>0$, $\left\langle X_{1}, X_{2}, \ldots, X_{k}\right\rangle$ is defined recursively for $k=1,2, \ldots$, by

$$
E\left\langle X_{1}, X_{2}, \ldots, X_{k}\right\rangle=0
$$

and

$$
\frac{\partial\left\langle X_{1}, X_{2}, \ldots, X_{k}\right\rangle}{\partial X_{i}}=\left\langle X_{1}, \ldots, X_{i-1}, \hat{X}_{i}, X_{i+1}, \ldots, X_{k}\right\rangle
$$

where $E$ means expectation (or mean) and $\hat{X}_{i}$ denotes the absence of the $X_{i}$ variable.

Example 5.1. The first two Wick products are

$$
\begin{aligned}
\left\langle X_{1}\right\rangle & =X_{1}-E X_{1}, \\
\left\langle X_{1}, X_{2}\right\rangle & =X_{1} X_{2}-X_{1} E X_{2}-X_{2} E X_{1}+2 E X_{1} E X_{2}-E X_{1} X_{2} .
\end{aligned}
$$

The Appell polynomials $P_{n}(x)$ are then defined by

$$
P_{X, n}(X)=P_{n}(X)=\underbrace{\langle X, \ldots, X\rangle}_{n \text { times }} .
$$

Example 5.2. Denoting $m_{1}=E X=0$ and $m_{i}=E X^{i}, i=2, \ldots$, we have:

$$
\begin{aligned}
& P_{0}(x)=1, \\
& P_{1}(x)=x \\
& P_{2}(x)=x^{2}-m_{2}, \\
& P_{3}(x)=x^{3}-m_{3}-3 m_{2} x \\
& P_{4}(x)=x^{4}-10 m_{2} x^{3}-10 m_{3} x^{2}+5 x\left(6 m_{2}^{2}-m_{4}\right), \\
& P_{5}(x)=x^{5}-10 m_{2} x^{3}-10 m_{3} x^{2}+5 x\left(6 m_{2}^{2}-m_{4}\right)+20 m_{2} m_{3}-m_{5} .
\end{aligned}
$$

Remark 5.1. If $X \sim N(0,1)$ (the Gaussian random variable with mean equal to 0 and variance equal to 1 ), then we get the familiar Hermite polynomials. But in general, Appell polynomials are not necessarily orthogonal polynomials.

Appell polynomials $P_{n}(x) ; n \in \mathbb{N}$ are also characterized by the two conditions

- $P_{n}(x)$ is a polynomial of degree $n$,

- $\frac{\mathrm{d}}{\mathrm{d} x} P_{n}(x)=n P_{n-1}(x)$.

Interesting examples are furnished by the shifted moment sequence

$$
P_{n}(x)=\int_{-\infty}^{\infty}(x+y)^{n} \mu(\mathrm{d} y),
$$

where $\mu$ is a probability measure on $\mathbb{R}$ with all moments finite. Of course, this includes in particular the Hermite polynomials for the Gaussian case. In [14] the 


\section{Henkel et al.}

probabilistic interpretation of Appell polynomials is used to define their analog on Lie groups where, in general, they are no longer polynomials. For this reason they are called Appell systems.

\section{Appell Systems of the Algebra $\mathfrak{a l t}_{1}$}

Appell systems of the Schrödinger algebra $\mathfrak{s c h}_{1}$ have been investigated in [11] but the algebra $\mathfrak{a l t}_{1}$ requires a specific study.

Referring to the decomposition (4), we specialize variables, writing $V_{1}, V_{2}, B_{1}, B_{2}$ for $A_{1}, A_{2}, A_{5}, A_{6}$ respectively. Basic for our approach is to calculate $e^{B_{1} Y_{-1}+B_{2} X_{-1}} e^{V_{1} Y_{1}+V_{2} X_{1}}$. We get

$$
B_{1} Y_{-1}+B_{2} X_{-1}=\left(\begin{array}{cccc}
0 & 0 & 0 & 0 \\
-B_{2} & 0 & -B_{1} & 0 \\
0 & 0 & 0 & 0 \\
0 & 0 & -B_{2} & 0
\end{array}\right), \quad V_{1} Y_{1}+V_{2} X_{1}=\left(\begin{array}{cccc}
0 & V_{2} & 0 & V_{1} \\
0 & 0 & 0 & 0 \\
0 & 0 & 0 & V_{2} \\
0 & 0 & 0 & 0
\end{array}\right)
$$

and finally:

$$
e^{B_{1} Y_{-1}+B_{2} X_{-1}} e^{V_{1} Y_{1}+V_{2} X_{1}}=\left(\begin{array}{cccc}
1 & V_{2} & 0 & V_{1} \\
-B_{2} & 1-B_{2} V_{2} & -B_{1} & -B_{2} V_{1}-B_{1} V_{2} \\
0 & 0 & 1 & V_{2} \\
0 & 0 & -B_{2} & 1-B_{2} V_{2}
\end{array}\right)
$$

Proposition 6.1. In coordinates of the second kind, we have the Leibniz formula

$$
\begin{aligned}
g\left(0,0,0,0, B_{1}, B_{2}\right) g\left(V_{1}, V_{2}, 0,0,0,0\right)=g\left(A_{1}, A_{2}, A_{3}, A_{4}, A_{5}, A_{6}\right) \\
=g\left(\frac{B_{1} V_{2}^{2}+V_{1}}{\left(1-B_{2} V_{2}\right)}, \frac{V_{2}}{\left(1-B_{2} V_{2}\right)},-2 \frac{B_{1} V_{2}+B_{2} V_{1}}{\left(1-B_{2} V_{2}\right)}\right. \\
\\
\left.\ln \left(1-B_{2} V_{2}\right), \frac{B_{1}-2 B_{1} B_{2} V_{2}-B_{2}^{2} V_{1}}{\left(1-B_{2} V_{2}\right)^{2}}, \frac{B_{2}}{\left(1-B_{2} V_{2}\right)}\right) .
\end{aligned}
$$

Now we are ready to construct the representation space and basis - the canonical Appell system. To start, define a vacuum state $\Omega$. The elements $Y_{1}, X_{1}$ of $\mathfrak{P}$ can be used to form basis elements

$$
|j k\rangle=Y_{1}^{j} X_{1}^{k} \Omega, \quad j, k \geq 0
$$

of a Fock space $\mathfrak{F}=\operatorname{span}\{|j k\rangle\}$ on which $Y_{1}, X_{1}$ act as raising operators, $Y_{-1}, X_{-1}$ as lowering operator and $Y_{0}, X_{0}$ as multiplication with the constants $\gamma, x$ (up to the 
sign) correspondingly. That is,

$$
\begin{aligned}
Y_{1} \Omega & =|10\rangle, X_{1} \Omega=|01\rangle, \\
Y_{-1} \Omega & =0, X_{-1} \Omega=0, \\
Y_{0} \Omega & =-\gamma|00\rangle, X_{0} \Omega=-x|00\rangle .
\end{aligned}
$$

The goal is to find an abelian subalgebra spanned by some self-adjoint operators acting on the representation space, just constructed. Such a two-dimensional subalgebra can be obtained by an appropriate "turn" of the plane $\mathcal{P}$ in the Lie algebra, namely via the adjoint action of the group element formed by exponentiating $X_{-1}$. The resulting plane, $\mathcal{P}_{\beta}$ say, is abelian and is spanned by

$$
\begin{aligned}
& \bar{Y}_{1}=e^{\beta X_{-1}} Y_{1} e^{-\beta X_{-1}}=Y_{1}-2 \beta Y_{0}+\beta^{2} Y_{-1}, \\
& \bar{X}_{1}=e^{\beta X_{-1}} X_{1} e^{-\beta X_{-1}}=X_{1}-2 \beta X_{0}+\beta^{2} X_{-1}
\end{aligned}
$$

Next we determine our canonical Appell systems. We apply the Leibniz formula (6.3) with $B_{1}=0, B_{2}=\beta, V_{1}=z_{1}, V_{2}=z_{2}$ and use (6.5). This yields

$$
\begin{aligned}
e^{z_{1} \bar{Y}_{1}} e^{z_{2} \bar{X}_{1}} \Omega & =e^{\beta X_{-1}} e^{z_{1} Y_{1}} e^{z_{2} X_{1}} e^{-\beta X_{-1}} \Omega=e^{\beta X_{-1}} e^{z_{1} Y_{1}} e^{z_{2} X_{1}} \Omega \\
& =e^{\frac{z_{1} Y_{1}}{\left(1-\beta z_{2}\right)^{2}}} e^{\frac{z_{2} X_{1}}{\left(1-\beta z_{2}\right)}} e^{\frac{2 \gamma \beta z_{1}}{\left(1-\beta z_{2}\right)}}\left(1-\beta z_{2}\right)^{-2 x} \Omega .
\end{aligned}
$$

To get the generating function for the basis $|j k\rangle$ set in Eq. (6.7)

$$
v_{1}=\frac{z_{1}}{\left(1-\beta z_{2}\right)^{2}}, \quad v_{2}=\frac{z_{2}}{\left(1-\beta z_{2}\right)} .
$$

Substituting throughout, we have

Proposition 6.2. The generating function for the canonical Appell system $|j k\rangle=$ $Y_{1}^{j} X_{1}^{k} \Omega i s$ :

$$
\begin{aligned}
e^{v_{1} Y_{1}+v_{2} X_{1}} \Omega= & \exp \left(y_{1} \frac{v_{1}}{\left(1+\beta v_{2}\right)^{2}}\right) \exp \left(y_{2} \frac{v_{2}}{\left(1+\beta v_{2}\right)}\right) \\
& \times \exp \left(-\frac{2 \gamma \beta v_{1}}{\left(1-\beta v_{2}\right)}\right)\left(1+\beta v_{2}\right)^{-2 x} \Omega
\end{aligned}
$$

where we identify $\bar{Y}_{1} \Omega=y_{1} \cdot 1$ and $\bar{X}_{1} \Omega=y_{2} \cdot 1$ in the realization as function of $y_{1}, y_{2}$.

Remark 6.1. With $v_{1}=0$, we recognize the generating function for the Laguerre polynomials, while $v_{2}=0$ reduces to the generating function of a standard Appell system. 


\section{Coherent States and Leibniz Function}

Now we define an inner product such that

$$
Y_{1}^{\dagger}=\beta^{2} Y_{-1}, \quad X_{1}^{\dagger}=\beta^{2} X_{-1} .
$$

In such a way the operators (6.6) are extended to self-adjoint ones on appropriate domains. For simplicity we take $\beta=1$ and define the two-parameter family of coherent states.

$$
\Psi_{V}=\Psi_{V_{1}, V_{2}}=e^{V_{1} Y_{1}} e^{V_{2} X_{1}} \Omega
$$

The Leibniz function is defined as inner product of coherent states

$$
\begin{aligned}
\mathcal{Y}_{B V} & =\left\langle\Psi_{B}, \Psi_{V}\right\rangle=\left\langle\Omega, e^{B_{1} Y_{-1}} e^{B_{2} X_{-1}} e^{V_{1} Y_{1}} e^{V_{2} X_{1}} \Omega\right\rangle \\
& =\left(1-B_{2} V_{2}\right)^{-2 x} \exp \left(\frac{2 \gamma\left(B_{1} V_{2}+B_{2} V_{1}\right)}{\left(1-B_{2} V_{2}\right)}\right) .
\end{aligned}
$$

Here we use the result (6.3) and a normalization $\langle\Omega, \Omega\rangle=1$ is understood. Further consideration shows that one can recover the raising and lowering operators as elements of the Lie algebra acting on the Hilbert space with basis consisting of the canonical Appell systems.

The remarkable fact is that the Lie algebra can be reconstructed from the Leibniz function $\mathcal{Y}_{B V}$. Really, differentiation with respect to $V_{1}$ brings down $Y_{1}$ acting on $\Psi_{V}$, while differentiation with respect to $B_{1}$ bring down $Y_{1}$ acting on $\Psi_{B}$ which moves across the inner product as $Y_{-1}$ acting on $\Psi_{V}$. Similarly for $X_{1}$ and $X_{-1}$. We thus introduce creation operators $\mathcal{R}_{i}$ and annihilation operators $\mathcal{V}_{i}$, satisfying $\left[\mathcal{V}_{i}, \mathcal{R}_{i}\right]=\delta_{i g} I$. For $\mathfrak{a l t}_{1}$, we identify $Y_{1}=\mathcal{R}_{1}, X_{1}=\mathcal{R}_{2}$. Note, however, that $\mathcal{V}_{1}$ is not adjoint of $\mathcal{R}_{1}$, nor $\mathcal{V}_{2}$ of $\mathcal{R}_{2}$. Bosonic realization of the respective adjoints $Y_{-1}, X_{-1}$, we want to determine now. One method is the following. When the explicit form of the Leibniz function $\mathcal{Y}_{B V}=\mathcal{Y}$ is known, one can (formally) write the partial differential equations for it. In our case they are

$$
\begin{aligned}
& \partial_{B_{1}} \mathcal{Y}=\left(V_{2}^{2} \partial_{V_{1}}+2 \gamma V_{2}\right) \mathcal{Y} \\
& \partial_{B_{2}} \mathcal{Y}=\left(V_{2}^{2} \partial_{V_{2}}+V_{1} V_{2} \partial_{V_{1}}+2 x V_{2}+2 \gamma V_{1}\right) \mathcal{Y} .
\end{aligned}
$$

Then, one interprets each multiplication by $V_{i}$ as the operator $\mathcal{V}_{i}$ and each differentiation by $V_{i}$ as the operator $\mathcal{R}_{i}$. This gives the following action of the operators $Y_{-1}, X_{-1}$ on polynomial functions of $Y_{1}$ and $X_{1}$ :

$$
\begin{aligned}
& Y_{-1}=2 \gamma \mathcal{V}_{2}+\mathcal{R}_{1} \mathcal{V}_{2}^{2} \\
& X_{-1}=\mathcal{R}_{2} \mathcal{V}_{2}^{2}+2 \mathcal{R}_{1} \mathcal{V}_{1} \mathcal{V}_{2}+2 \gamma \mathcal{V}_{1}+2 x \mathcal{V}_{2}
\end{aligned}
$$

From the commutation relations, we find $Y_{0}$ and $X_{0}$

$$
Y_{0}=-\mathcal{R}_{1} \mathcal{V}_{2}-\gamma, X_{0}=-\mathcal{R}_{1} \mathcal{V}_{1}-\mathcal{R}_{2} \mathcal{V}_{2}-x
$$


Using the more usual notation $a_{1}=\mathcal{V}_{1}, a_{2}=\mathcal{V}_{2}, a_{1}^{+}=\mathcal{R}_{1}, a_{2}^{+}=\mathcal{R}_{2}$ we have the following:

Proposition 7.1. The raising $a_{1}^{+}, a_{2}^{+}$and lowering $a_{1}, a_{2}$ operators appear as elements of the algebra $\mathfrak{a l t}_{1}$ in the following way:

$$
\begin{aligned}
Y_{1} & =a_{1}^{+}, \quad X_{1}=a_{2}^{+}, \\
Y_{0} & =-a_{1}^{+} a_{2}-\gamma, \quad X_{0}=-a_{1}^{+} a_{1}-a_{2}^{+} a_{2}-x, \\
Y_{-1} & =a_{1}^{+} a_{2}^{2}+2 \gamma a_{2}, \quad X_{-1}=a_{2}^{+} a_{1}^{2}+2 a_{1}^{+} a_{1} a_{2}+2 \gamma a_{1}+2 x a_{2} .
\end{aligned}
$$

The action on the Fock space, consisting of two parameter family of coherent state (7.2) with basis the canonical Appell systems with generated function (6.9) is as follows:

$$
\begin{aligned}
Y_{1}|j, k\rangle & =|j+1, k\rangle, \quad X_{1}|j, k\rangle=|j, k+1\rangle, \\
Y_{0}|j, k\rangle & =-k|j+1, k-1\rangle-\gamma|j, k\rangle, \quad X_{0}|j, k\rangle=-(j+k+x)|j, k\rangle, \\
Y_{-1}|j, k\rangle & =k(k-1)|j+1, k-1\rangle+2 \gamma|j, k-1\rangle, \\
X_{-1}|j, k\rangle & =k(k+2 j+2 x-1)|j, k-1\rangle+2 j \gamma|j-1, k\rangle .
\end{aligned}
$$

The natural involution on the algebra $Y_{1} \leftrightarrow Y_{-1}, X_{1} \leftrightarrow X_{-1}$, with substitutions $-a_{1} \leftarrow r,-a_{2} \leftarrow t,-a_{1}^{+} \leftarrow \partial_{r},-a_{2}^{+} \leftarrow \partial_{t}$ leads to the physical representation $(2.21)$.

\section{Concluding Remarks}

We have studied properties of the Poincaré or "altern" algebra $\mathfrak{a l t}_{1}$, nowadays also often referred to as "conformal galilean algebra", and of interest in connection with the ageing phenomenon in condensed-matter physics and in string-theory in the context of the AdS/CFT correspondence. In particular, we have shown that $\mathfrak{a l t}_{1}$ can be embedded in an infinite-dimensional Lie algebra and have discussed its relationship with the Virasoro and the Schrödinger-Virasoro algebras.

As for the representation-theory, we have systematically constructed the Casimir operators and have written down explicit matrix representations. Considerations of the Wick product has led us to the construction of the Appell systems of $\mathfrak{a l t}_{1}$ which are useful for the construction of coherent states.

A more general study of random walks and stochastic processes on $\mathfrak{a l t}_{1}$ is a challenging research project.

\section{Acknowledgment}

The authors have been supported by the EU Research Training Network HPRNCT-2002-00279. 


\section{References}

1. F. Avram and M. S. Taqqu, Noncentral limit theorems and Appell polynomials, Ann. Probab. 15 (1987) 767-775.

2. A. O. Barut, Conformal group $\rightarrow$ Schrödinger group $\rightarrow$ Dynamical group - the maximal kinematical group of the massive Schrödinger particle, Helv. Phys. Acta 46 (1973) 496-503.

3. F. Baumann, S. Stoimenov and M. Henkel, Local scale-invariances in the bosonic contact and pair-contact processes, J. Phys. A Math. Gen. 39 (2006) 4095-4118.

4. C. D. Boyer, R. T. Sharp and P. Winternitz, Symmetry-breaking interactions for the time dependent Schrödinger equation, J. Math. Phys. 17 (1976) 1439-1451.

5. A. J. Bray, Theory of phase-ordering kinetics, Adv. Phys. 43 (1994) 357-459.

6. G. Burdet, M. Perrin and P. Sorba, About the non-relativistic structure of the conformal algebra, Comm. Math. Phys. 34 (1973) 85-90.

7. R. Cherniha and M. Henkel, The exotic conformal Galilei algebra and nonlinear partial differential equations, J. Math. Anal. Appl. 369 (2010) 120-132.

8. L. Clavelli and P. Ramond, Group-theoretical construction of dual amplitudes, Phys. Rev. D 3 (1971) 988-990.

9. L. F. Cugliandolo, Dynamics of glassy systems, in Slow Relaxation and nonequilibrium Dynamics in Condensed Matter, Les Houches Session 77, July 2002, eds. J.-L. Barrat, J. Dalibard, J. Kurchan, M. V. Feigel'man (Springer, 2003).

10. C. Duval and P. A. Horváthy, Non-relativistic conformal symmetries and NewtonCartan structures, J. Phys. A: Math. Theor. 42 (2009) 465206.

11. P. Feinsilver, Y. Kocik and R. Schott, Representations of the Schrödinger algebra and appell systems, Prog. Phys. 52 (2004) 343-359.

12. P. Feinsilver and R. Schott, Algebraic Structures and Operator Calculus, Vol. 1: Representations and Probability Theory (Kluwer, 1993).

13. P. Feinsilver and R. Schott, Algebraic Structures and Operator Calculus, Vol. 3: Representations of Lie Groups (Kluwer, 1996).

14. P. Feinsilver and R. Schott, Appell systems on Lie groups, J. Th. Probab. 5 (1992) 251-281.

15. D. B. Fuks, Cohohomology of infinite-dimensional Lie algebras, in Monographs in Contemporary Soviet Mathematics, Consultants Bureau (Springer, 1986).

16. L. Guieu and C. Roger, L'algèbre et le groupe de Virasoro: Aspects géométriques et algébriques, généralisations, Publications CRM (Montreal Univ. Press, 2007).

17. P. Havas and J. Plebanski, Conformal extensions of the Galilei group and their relation to the Schrödinger group, J. Math. Phys. 19 (1978) 482-488.

18. M. Henkel, Schrödinger-invariance and strongly anisotropic critical systems, J. Stat. Phys. 75 (1994) 1023-1061.

19. M. Henkel, Phenomenology of local scale-invariance: From conformal invariance to dynamical scaling, Nucl. Phys. B 641 (2002) 405-486.

20. M. Henkel and J. Unterberger, Schrödinger-invariance and space-time symmetries, Nucl. Phys. B 660 (2003) 407-435.

21. M. Henkel and J. Unterberger, Supersymmetric extensions of Schrödinger-invariance, Nucl. Phys. B $\mathbf{7 4 6}$ (2006) 155-201.

22. M. Henkel, R. Schott, S. Stoimenov and J. Unterberger, On the dynamical symmetric algebra of ageing: Lie structure, representations and Appell systems, Quantum Probab. White Noise, 20 (2007) 233-240.

23. Eds. M. Henkel, M. Pleimling and R. Sanctuary, Ageing and the Glass Transition, Lecture Notes in Physics, Vol. 716 (Springer-Verlag, 2007). 
24. M. Henkel and M. Pleimling, Non-equilibrium Phase Transitions Vol. 2: Ageing and Dynamical Scaling Far from Equilibrium (Springer, 2010).

25. M. Henkel and S. Stoimenov, On non-local representations of the ageing algebra, Nucl. Phys. B 847 (2011) 612-627.

26. C. G. Jacobi, Vorlesungen über Dynamik (1842/43), 4. Vorlesung, in Gesammelte Werke, eds. A Clebsch und E. Lottner (Akademie der Wissenschaften, 1866/1884).

27. S. Lie, Über die Integration durch bestimmte Integrale von einer Klasse linearer partieller Differentialgleichungen, Arch. Math. Naturvidenskab. 6 (1881) 328.

28. J. Lukierski, P. C. Stichel and W. J. Zakrewski, Exotic galilean conformal symmetry and its dynamical realisations, Phys. Lett. A 357 (2006) 1-5; Accelaration-extended galilean symmetries with central charges and their dynamical realizations, Phys. Lett. B 650 (2007) 203-207.

29. U. Niederer, The maximal kinematical invariance group of the free Schrödinger equation, Helv. Phys. Acta 45 (1972) 802-810.

30. A. Picone and M. Henkel, Local scale-invariance and ageing in noisy systems, Nucl. Phys. B 688 (2004) 217-265.

31. C. Roger and J. Unterberger, The Schrödinger-Virasoro Lie group and algebra: From geometry to representation theory, Ann. Inst. H. Poincaré 7 (2006) 1477-1529.

32. S. Stoimenov and M. Henkel, Dynamical symmetries of semi-linear Schrödinger and diffusion equations, Nucl. Phys. B 723 (2005) 205-233.

33. J. Unterberger and C. Roger, The Schrödinger-Virasoro Algebra (Springer, 2011).

34. M. Virasoro, Subsidiary conditions and ghosts in dual-resonance models, Phys. Rev. D 1 (1970) 2933-2936. 